\title{
FEHDE UND DEUTSCHER ORDEN, VORNEHMLICH IM DEUTSCHEN REICH
}

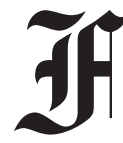

ehde ist in neueren Publikationen inzwischen wieder etwas in Frage gestellt worden. Nach Hans-Henning Kortüm könne mit der Fehde nur ein eng begrenztes Bedeutungsfeld abgedeckt werden. Er wendet sich vehement gegen die Darstellung Otto Brunners, der „Fehde“ damit zu erklären suchte, dass ein Ritter sein Recht nach allgemein akzeptierten Regeln selbst zu suchen habe. ${ }^{1}$ Was Brunner freilich verschwiegen oder zumindest kaum ausgeführt hat, ist die Tatsache, dass die Fehde führenden Adligen dafür sorgten oder zumindest dafür Sorge trugen, dass ihre Sicht der Dinge und damit auch ihre Rechtfertigungsgründe schriftlich festgelegt wurden, in Schiedssprüchen oder auch gelegentlich gerichtlichen Urteilen Eingang fanden. Ohne Schwierigkeiten lassen sich andere Argumente vor allem in städtischen Archiven finden, die das Unrecht der Fehdeführenden anprangerten. Kaufleute und Mitglieder der städtischen Führungsschichten, die am meisten unter den sogenannten Fehden zu leiden hatten, haben vielfach darauf verwiesen, dass bestimmte Regularien, auf die so viel Wert gelegt worden ist, gar nicht eingehalten worden seien. Diese Argumente wurden wiederum von meist adligen Fehdeführern bestritten. ${ }^{2}$ Es hing daher oft davon ab, auf

1 H.-H. Kortüm, Kriege und Krieger 500-1500, Stuttgart 2010, S. 70-74; vgl. auch G. Algazi, Herrengewalt und Gewalt der Herren im späten Mittelalter (Historische Studien 17), Frankfurt-New York 1996, S. $97 \mathrm{ff}$.

2 O. Brunner, Land und Herrschaft, Wien ${ }^{5} 1965$, S. 41-110; in dessen Sinn auch für Westfalen: R. Görner, Raubritter (Veröffentlichungen der Historischen Kommission für Westfalen 22; Geschichtliche Arbeiten zur westfälischen Landesforschung 18), Münster/Westfalen 1987, vor allem S. 173-281. Im Sinne Brunners für Köln: U. Lindgren, Kölner Fehden als Problem von Verwaltung und Verfassung (1370-1400), Jahrbuch des Kölnischen Geschichtsvereins 54 (1983), S. 1 ff. Dagegen spricht H. Ulmschneider, Götz von Berlichingen, Sigmaringen 1974, S. 92 f., unumwunden von „Raub“ hinsichtlich der Fehden des Götz von Berlichingen. Ferner: R. Sablonier, Rittertum, Adel und Kriegswesen im Spätmittelalter, in: Das ritterliche Turnier im Mittelalter, hrsg. v. J. Fleckenstein (Veröffentlichungen des Max-Planck-Instituts für Geschichte 
welche Darstellung sich ein Historiker stützte, wenn er eine Fehde für rechtmäßig erklärte oder im Gegenteil als unrechtmäßig bezeichnete. ${ }^{3}$ Zudem war es häufig eine Frage der Politik, ob sich ein bestimmter Gesichtspunkt durchsetzen ließ oder nicht, ob ein fehdeführender Adliger als ein Unrecht oder Raub begehender oder als sein Recht suchender und durchsetzender Mann von seinen Zeitgenossen angesehen wurde, ob ihn ein hochmögender Fürst beschützte oder die Hand von ihm gezogen hatte, weil sich dieser Landesherr von der Rücknahme seiner Huld Vorteile erhoffen konnte. ${ }^{4}$ Ferner scheint es sehr auf die Region angekommen zu sein, aus der der jeweilige Historiker seine wesentlichen Quellen und Anschauungen bezogen hat. ${ }^{5}$ Möglicherweise herrschten in Westfalen und dem Rheinland andere Bedingungen als etwa in Süddeutschland mit den doch eher zerklüfteten Herrschaftsbereichen. Hinzu traten die sogenannten Stellvertreterfehden, die Adlige für einen anderen ausfochten. Gerade diese Form der Fehden fand selten die Unterstützung der Städte und deren Räten. Im übrigen trafen die Fehden nicht nur die Kaufleute und die Städte, sondern ebenso Klöster und eben den Deutschen Orden und seine Balleien. ${ }^{6}$ Das alles sei vorausgeschickt, weil die Diskussion über Fehden in der deutschen Geschichtswissenschaft und darüber hinaus inzwischen an Schärfe zugenommen hat und teilweise geradezu vergiftet ist.

Fehden sollten den Deutschen Orden und seine Mitglieder eigentlich nicht beunruhigen. Denn, wie die Regel um 1250 festlegte, habe sich ein Ordensmitglied der Freiheiten und Privilegien, wie Päpste sie geistlichen Institutionen erteilt hätten, zu erfreuen. Falls jemand ihnen diese Privilegien streitig machen sollte, hätten die Ordensbrüder sie vor weltlichen Gerichten einzuklagen. Sollten sie dort

80), Göttingen 1985, S. 560 f.; N. Ohler, Krieg und Frieden im Mittelalter (Beck'sche Reihe 1226), München 1997, besonders S. 299 ff.; W. Rösener, Zur Problematik des spätmittelalterlichen Raubrittertums, in: Festschrift für Berent Schwineköper zu seinem 70. Geburtstag, hrsg. v. H. Maurer, H. Patze, Sigmaringen 1982, S. 469-488; K. Andermann, Raubritter, in: Lexikon des Mittelalters, Bd. 7, München 1995, Sp. 474 f., zusammenfassend in seiner Abwehr des Begriffs „Raubritter“ und der Anerkennung der Fehde als Instruments des Adels zur Durchsetzung seines Rechts. Dazu abwägend: K. Graf, Feindbild und Vorbild. Bemerkungen zur städtischen Wabrnehmung des Adels, Zeitschrift für die Geschichte des Oberrheins 141 (1993), S. 121-154.

3 Vgl. auch H. Boockmann, Fehde, Fehdewesen, in: Lexikon des Mittelalters, Bd. 4, München-Zürich 1989, Sp. 331-334.

4 Zu Recht weist Graf (wie Anm. 2), S. 129 und öfter darauf hin, dass „einseitige Schuldzuweisungen" abzulehnen seien. Ob man seinen Bewertungen folgen kann, sei dahin gestellt. Vgl. auch Algazi (wie Anm. 1), S. 138 ff., 140 ff., 154 ff.

5 Vgl. Rösener (wie Anm. 2), S. 477 ff.

6 Vgl. Ebd., S. 471 f., der vor allem auf Westfalen gegen Brunner und seine vorwiegend aus süddeutschen und österreichischen Quellen bezogenen Argumente hinweist. 
kein Recht erhalten, sei es ihnen verboten, rücksichtslos vorzugehen. ${ }^{7}$ In den wenig später zu datierenden Gesetzen des Ordens wurde als schwere Schuld gewertet, wenn ein Ordensbruder einen Christen verwundet hätte, so dass er blutete ${ }^{8}$, oder, wie man hinzu fügen könnte, wenn er ihn gar erschlüge. Als Strafe drohte dem Delinquenten die Jahrbuße, von der der Hochmeister in der Regel nichts erlassen konnte. ${ }^{9}$ Die Jahrbuße war erniedrigend und demütigend. Denn der Bestrafte hatte während des folgenden Jahres nicht mehr in einem Saal mit seinen Mitbrüdern, sondern bei den Sklaven zu schlafen. Er sollte sein Kreuz auf dem Mantel verlieren, mit den Dienern, getrennt von den Mitbrüdern, auf dem Boden essen und trinken. Ferner hatte er zu fasten und an allen Sonntagen vom Priesterbruder eine Züchtigung entweder in der Ordenskirche oder auch im Kapitel zu empfangen. ${ }^{10}$

In einem Männerorden kam es wohl häufiger vor, dass Auseinandersetzungen mit Waffengewalt ausgetragen worden sind. Jedenfalls bestimmte Hochmeister Luther von Braunschweig (1331-1335) wohl als Reaktion auf eine vorausgegangene Straftat, dass jemand, der einen anderen Ordensbruder erschlüge, mit Gefängnis bestraft werden solle. Diese Strafe dürfe niemand außer dem Hochmeister mit Zustimmung des Kapitels aufheben und den Delinquenten aus dem Gefängnis entlassen. ${ }^{11}$ Luthers Nachfolger Dietrich von Altenburg (1335-1341) erklärte, dass ein Bruder, der einen anderen mit Messer, Schwertern oder anderen Waffen verletze oder gar töte oder auch nur damit drohe, mit eisernen Fesseln gefangen gehalten und dem Hochmeister vorgeführt werde. ${ }^{12}$ Aus der Wiederholung und teilweisen Verschärfung der Vorschrift ist zu schließen, dass dergleichen Vorkommnisse nicht selten gewesen zu sein scheinen.

Allerdings war die Notwehr erlaubt. In bestimmten Fällen durfte sich der Ritterbruder eines tätlichen Angriffs erwehren. Wenn die Gesetze ausdrücklich

7 Vgl. die Beilagen in: Aachener Stadtrechnungen aus dem XIV. Jahrhundert, hrsg. v. J. Laurent, Aachen 1866, S. 78 ff. Dazu: K. Militzer, Stadt und Fehde: Das Kölner Beispiel, Fasciculi archaeologiae historicae 15 (2003) (Le convoi militaire, hrsg. v. T. Poklewski-Koziełt), S. $87 \mathrm{ff}$.

8 M. Perlbach, Die Statuten des Deutschen Ordens nach den ältesten Handschriften, Halle/Salle 1890, S. 30 f.: Regel 3; dazu K. Militzer, Von Akkon zur Marienburg - Verfassung, Verwaltung und Sozialstruktur des Deutschen Ordens 1190-1309 (Quellen und Studien zur Geschichte des Deutschen Ordens 56; Veröffentlichungen der Internationalen Historischen Kommission zur Erforschung des Deutschen Ordens 9), Marburg 1999, S. 48 f.

9 Perlbach, Statuten (wie Anm. 8), S. 83: Gesetze 38 (1).

10 Militzer, Von Akkon (wie Anm. 8), S. 92; G. Schmidt, Die Handhabung der Strafgewalt gegen Angehörige des Deutschen Ritterordens (Beihefte zum Jahrbuch des Albertus-Universität zu Königsberg/Pr. 4), Kitzingen/Main 1954, S. 100 f.

11 Perlbach, Statuten (wie Anm. 8), S. 85 f.

12 Ebd., S. $149 \$ 6$. Er erneuerte damit eine bereits getroffene Bestimmung des Hochmeisters Gottfried von Hohenlohe von 1297: ebd., S. $144 \$ 2$. 
bestimmten, dass niemand sich oder seine Habe gegen einen Christen mit Waffengewalt schützen dürfe, indem er im Zorn oder mit Vorsatz handle ${ }^{13}$, ist daraus im Umkehrschluss zu folgern, dass er sehr wohl bei kühlem Kopf sich und seine Habe verteidigen dürfe. Es ist nun aber strittig, ob Ritterbrüder in den Balleien des Reiches während ihrer Ausritte jemals Schwerter oder andere Waffen getragen haben. Darüber ist jedenfalls noch nicht viel bekannt. Es dürfte auch schwer sein, einschlägige Quellen zu finden, da über Ausritte während des Mittelalters kein Buch geführt, geschweige denn festgehalten worden ist, ob der Ausreitende eine Waffe bei sich gehabt habe oder nicht. Diese Überlegung erschwert wiederum eine Abschätzung dessen, ob ein Ritterbruder in den Balleien jemals in Notwehr habe handeln können. Denn die Regeln, Gesetze und Gewohnheiten waren vor allem für diejenigen gedacht, die an der Front in Palästina und später auch in Preußen und in Livland eingesetzt worden waren, also im 13. und teilweise noch im 14. Jahrhundert in Feindesland lebten. Später hatten sie dort die Grenzen gegen Ungläubige oder Schismatiker zu schützen. Von ihnen konnte man erwarten, dass sie bei Ausritten eine Waffe trügen und sich in Notwehr schützen würden. ${ }^{14}$

In den Balleien versuchten die Amtsträger des Ordens deshalb, Schutz und Schirm bei König, Kaiser und Reich oder den geistlichen und weltlichen Landesherren und den Rittervereinigungen wie der Gesellschaft vom Jörgenschild zu suchen. ${ }^{15}$ Schutz und Schirm waren aber nicht umsonst zu haben. Dem König oder Kaiser waren Dienste zu leisten. Die Amtsträger, vor allem der Deutschmeister und auch der Landkomtur von Franken, wurden Räte des Reichsoberhauptes. ${ }^{16}$ Dienste verschiedener Art verlangten sowohl geistliche als auch weltliche Landesherren. Die Gesellschaft vom Jörgenschild forderte vom Deutschen Orden in erster Linie Beiträge, auch wenn das in der einschlägigen Literatur vielfach nicht angegeben ist. ${ }^{17}$ Jedoch bot die Gesellschaft Schutz vor Fehden und zwang Gegner des Deutschen Ordens vor Schiedsgerichte. ${ }^{18}$ Solche Schiedsgerichte sind viel-

13 Ebd., S. $151 \$ 24$.

14 Ebd., S. 83, Gesetz $38 \$ 1$ : [...] irato animo vel ex proposito [...]; oder in der Übersetzung: [...] in zorne oder mit bedâhtem mûte [...].

15 K. Militzer, Die Einbindung des Deutschen Ordens in die süddeutsche Adelswelt, in: Ritterorden und Region, hrsg. v. Z. H. Nowak (Ordines militares. Colloquia Torunensia Historica VII), Toruń 1995, S. 149-154.

16 Vgl. R. ten Haaf, Deutschordensstaat und Deutschordensballeien (Göttinger Bausteine zur Geschichtswissnschaft 5), Göttingen 2 1951, S. 64 ff.

17 Vgl. statt vieler die Angaben: Andreas Ranft und Sonja Zielke-Dünnebeil in: Ritterorden und Adelsgesellschaften im spätmittelalterlichen Deutschland, hrsg. v. H. Kruse, W. Paravicini, A. Ranft (Kieler Werkstücke, Reihe D: Beiträge zur europäischen Geschichte des späten Mittelalters 1), Frankfurt/Main u. a. 1991, S. 202-217; dazu: Militzer, Einbindung (wie Anm. 16), S. 153. 
fach schon seit dem 13. Jahrhundert bezeugt. Beispielsweise schlichtete im Jahr 1293 die Gräfin von Kleve zusammen mit ihrem Sohn Dietrich einen Streit zwischen der Kommende Koblenz und Johann von Are und dessen Angehörigen. ${ }^{19}$

Die Abwendung einer Fehde ist freilich nicht immer gelungen. Wir wollen uns in diesem Zusammenhang eine Fehde aus dem Rheinland genauer ansehen. Als am 23. September 1453 ein rheinischer Ritter namens Johann von Hochstaden oder Hoisten wegen seiner Güter in Noithausen bei Grevenbroich dem Deutschordenshaus in Koblenz einen Fehdebrief übergeben ließ, wurde auch festgestellt, dass Johanns Frau zwei Tage vorher, nämlich noch am 21. September, an einer Hochzeitsfeier mit den späteren Fehdegegnern teilgenommen und der Braut ein Geldgeschenk verehrt habe. Der Fehdebrief datiert vom 23. September, wie auch allerseits anerkannt worden ist. Er sei aber erst am Abend des darauf folgenden Tages, dem 24. September, im Deutschordenshaus in Koblenz eingetroffen. Bereits am frühen Morgen des 27. September habe Johann mit seinen Helfern die Fehde begonnen. Nach Auffassung des Deutschen Ordens habe ein Ordensangehöriger keine Möglichkeit gehabt, seine Leute in Elsen bei Grevenbroich zu informieren, damit sie hätten Vorsorge treffen können. ${ }^{20}$ Immerhin liegt Elsen etwa $150 \mathrm{~km}$ von Koblenz in Richtung Norden entfernt. Selbst bei gutem Willen hätte der Orden diese Entfernung mit vorzüglichen Pferden kaum rechtzeitig an den beiden Tagen, nämlich dem 25. und 26. September, bewältigen können. Man braucht nur nachzurechnen. Am 24. abends war nicht mehr viel zu machen, so dass erst am folgenden Tag mit der Überbringung der Botschaft begonnen werden konnte. Ein Pferd konnte allenfalls $60 \mathrm{~km}$ pro Tag bewältigen. Das aber hätte nicht gereicht, um die Menschen in Elsen vor dem Angriff in der Frühe am 27. des Monats zu warnen. Der Orden hat die Schäden an seinen Untertanen in Elsen durch sein Schöffengericht bestätigen und die entsprechenden Aussagen mit Hilfe von Notariatsinstrumenten festhalten lassen. Daher wissen wir darüber Bescheid. Jedoch stammen die Schriftstücke alle aus dem Interessenskreis des Ordens selbst. Von dem Ritter sind keine Nachrichten oder Stellungnahmen überliefert. Immerhin wird man fragen können, ob es sich um eine gerechte Fehde gehandelt habe. Nach Brunners Definition und der ihm folgenden Interpretatoren sehr wohl. Allerdings könnte man einwenden, dass die Dreitagesfrist nicht eingehalten und die länger andauernde

gen der Gesellschaft für fränkische Geschichte, Reihe IX: Darstellungen aus der fränkischen Geschichte 39), Neustadt/Aisch 1991, S. 347.

19 Historisches Archiv der Stadt Köln (künftig: HAStK), Katharinen U 1 2/133. Weitere Belege allein aus diesem Bestand: 2/186 (1305), 1/226 (1320); 1/364 (1357), 3/471 (1389), 3/584 (1426), 1/593 (1430), 1/641 (1447), 1/643 (1449), 2/644, 1/645 (beide 1450), 1/653 (1451), $1 / 733$ (1456), 3/746 (1481), 1/756 (1484), 3/770 (1488).

20 HAStK, Katharinen, U 2/656, 2/657, 3/661, 3/663, 3/668. 
Feindschaft zwischen beiden Parteien nicht gegeben gewesen sei. ${ }^{21}$ Darauf hatte der Orden mit Nachdruck hingewiesen. In der Sache ging es eher um einen uns heute als Nebensächlichkeit erscheinenden Vorgang. Denn der Ritter klagte darum, dass seine Leute von ihrem eigenen Gerichtsboten vor das Schöffengericht in Elsen geladen würden, während der Orden darauf bestand, dass auch solche Leute von dem Elsener Gerichtsboten vor das Gericht in Elsen geladen würden.

Wie der Streit oder die Fehde schließlich ausgegangen ist, bleibt im Dunkeln. Wahrscheinlich hat Johann von Hochstaden nach einem Schiedsgerichtsverfahren klein beigegeben müssen. Jedenfalls hat er sich mit seinen Forderungen nicht durchsetzen können, wie aus anderen Urkunden hervorzugehen scheint. Wie Johann von Hochstaden ist es vielen anderen Adligen in ihren Auseinandersetzungen mit dem Orden ergangen. Sie haben sich meist einem schiedsrichterlichen Verfahren beugen müssen, weil ein mächtiger Landesfürst die Hand über den Orden gehalten hat. Es mag sich daher erübrigen, weitere derartige Fehden, die sich vor allem im 15. Jahrhundert häuften, im einzelnen vorzustellen.

Eine andere frühere Fehde Hermanns, des Bijssers, mit dem Ordenshaus Koblenz und dem gesamten Orden, wie es heißt, endete 1346 mit einem Schiedsspruch und einer Sühne für den Fehdeführer und dessen Helfer. Wiederum ging es um Elsen. Hermann und seine Mitstreiter wurden verpflichtet, ihr Recht vor dem Schöffengericht in Elsen zu suchen und ihre Ansprüche nicht über die Gerichtsgrenzen hinaus zu verfolgen. Eingeschlossen in die Sühne waren auch die Schöffen von Elsen wie ein Elsener Bürger namens Johann von Blatzheim. ${ }^{22}$ Eine analoge Fehde wurde 1402 durch ein Schiedsgericht geschlichtet. Sie richtete sich gegen die Kölner Kommende und deren Hof bei Nievenheim. ${ }^{23}$

Wenn man die Urkunden der Kölner Katharinenkommende näher untersucht, kommt man zu der Feststellung, dass sich eine Entwicklung abzeichnen könnte. Freilich ist diese Tendenz noch anhand anderer Urkundenbestände zu verifizieren. Nach der Kölner Überlieferung sieht es so aus, als ob zu Beginn des 14. Jahrhunderts Fehden, die auch als solche bezeichnet worden sind, im Zusammenhang mit anderen Unglücksfällen wie Hagel, Raub, Brand und dergleichen, genannt worden sind, wenn es darum ging, Höfe oder größere Grundstücke zu verpachten. In solchen Fällen wurde der Pächter verpflichtet, auch dann die Pacht

21 Auf beide Kriterien legt Rösener (wie Anm. 2), S. 481 ff. großen Wert als Unterscheidung zwischen Fehde und Überfall und rechter bzw. unrechter Fehde.

22 HAStK, Katharinen, U 1/322.

23 HAStK, Katharinen, U 1/509; vgl. das Regest: Gohr, Nievenheim, Straberg. Quellen zur Geschichte des Amtes Nievenheim, seiner Bewohner und Siedlungen, Tl. 1, hrsg. v. W. Lorenz im Auftrag des Amtes Nievenheim, nach Vorarbeiten v. H. Breuer, A. \& H. Vogel (Inventare nichtsstaatlicher Archive 16), Köln 1973, S. 160, Nr. 402, allerdings mit falschem Datum. 
zu entrichten, wenn beispielsweise eine Fehde ausgebrochen sei. ${ }^{24}$ In der zweiten Hälfte des Jahrhunderts hat der Orden in diesem Zusammenhang meist nur von göttlicher oder höherer und menschlicher Gewalt gesprochen, ohne die Fehde ausdrücklich zu erwähnen. ${ }^{25}$ Das trifft auch noch für das erste Jahrzehnt des 15. Jahrhunderts zu. ${ }^{26}$ Von dem Zeitpunkt an ist die Fehde wieder wie schon im 14. Jahrhundert in die Pachtverträge aufgenommen worden. ${ }^{27}$ Seit 1488 jedoch, als Werner Spieß von Büllesheim Komtur von Koblenz war, ${ }^{28}$ wurde in diesen Verträgen zwischen den einfachen Fehden und den „Feinden des Landesherren“ unterschieden. ${ }^{29}$ Allerdings ist in einem Pachtvertrag von 1494 von den „Feinden des Landesherrn" keine Rede mehr..$^{30}$ Möglicherweise hat der Orden für eine besondere Situation Vorsorge getroffen. Jedoch bleibt das offen, zumal ein eindeutiger Hinweis auf einen solchen Zusammenhang fehlt. Immerhin sei auf die Erbstreitigkeiten der rheinischen und burgundischen Fürstenhäuser am Ende des 15. Jahrhunderts hingewiesen. ${ }^{31}$

Ob sich die Beobachtung, die ich anhand des Kölner Urkundenmaterials vorgestellt habe, auf andere Gegenden ausweiten lässt, kann ich momentan nicht beurteilen. ${ }^{32}$ Dazu bedürfte es eingehenderer Untersuchungen der urkundlichen Hinterlassenschaften des Ordens in anderen Regionen. Jedoch ist zu beobachten, dass die Schöffen- und andere Gerichte in Köln, Bonn, Bad Godesberg, Lechenich und in weiteren Orten stark genug waren, dem Orden dessen Recht zu gewähren und auch durchzusetzen. Mit dem Hinweis auf die Kölner Dokumente mag es sein Bewenden haben. Dennoch wird man generell sagen dürfen, dass sich die Fehden adliger Grundbesitzer gegen den Orden bis zum 15. Jahrhundert hinein gehäuft haben. Das ist wohl nicht nur auf die vermehrte Schriftlichkeit der Zeit zurückzuführen. ${ }^{33}$ Dagegen blieben Fehden der Landesfürsten eher eine Seltenheit, zu-

24 HAStK, Katharinen, U 3/223 (1319), 2/231 (1321), 1/319 (1345).

25 HAStK, Katharinen, U 1/433 (1371), 2/447 (1377), 1/450 (1382), 3/485 (1395).

26 HAStK, Katharinen, U 2/508 (1402), 2/510 (1404), 2/526 (1408); 3/538 (1410).

27 HAStK, Katharinen, U 2/508 (1402), $3 / 584$ (1426), 2/615 (1438), 2/659 (1454), 1/669 (1455), 2/688 (1463), 2/703 (1463), 2/716 (1469). Da es sich im letzteren Fall um die Verpachtung einer Wassermühle handelte, ist der Falle der Wassernot hinzugenommen worden.

28 H. Limburg, Die Hochmeister des Deutschen Ordens und die Ballei Koblenz (Quellen und Studien zur Geschichte des Deutschen Ordens 8), Bad Godesberg 1969, S. 189.

29 HAStK, Katharinen, U 2/771 (1488), 2/775 (1489).

30 HAStK, Katharinen, U 2/781 (1494).

31 Allgemein vgl. M. Prietzel, Krieg im Mittelalter, Darmstadt 2006, S. 181 f.

32 Vgl. aus dem benachbarten Moselraum die Andeutungen bei W. Reichert, Hominum dura cervix: Agrarische Konflikte und Konfliktlösungen an der Mosel und in der Eifel während des hohen Mittelalters, Rheinische Vierteljahrsblätter 75 (2011), S. 79 ff.

33 So auch Görner (wie Anm. 2), S. 274. 
mal sich der Orden gerade diese Fürsten geneigt gemacht und deren Autorität als Schiedsrichter in Anspruch genommen hat.

Allerdings fanden Fehden gegen den Orden nicht regelmäßig statt, sondern bildeten die Ausnahme. Streitpunkte hat der Orden oft durch Schiedsleute oder eine Schiedsgerichtsbarkeit beheben lassen. Als 1477 Hans von Degenberg, ein Erbhofmeister und einflussreicher Mann, eine Fehde gegen den Orden ansagte, weil die Untertanen des Ordensamts Postbauer in Franken sie ihm aufgezwungen hätten, hat der Landkomtur von Franken den Markgrafen von Brandenburg-Ansbach um einen Schiedsspruch angerufen. Der Markgraf fällte ein Urteil, das der Erbhofmeister schließlich angenommen hat. ${ }^{34}$

Diese Schiedsgerichtsbarkeit kommt in ihrer schriftlichen Form erst seit dem 12. Jahrhundert vor. Sie mag schon früher mündlich vereinbart worden sein. Jedoch wissen wir darüber wenig. ${ }^{35}$ Für die Balleien des Deutschen Ordens spielte sie jedenfalls vor allem im 14. und 15. Jahrhundert eine bedeutende Rolle, wie viele Urkunden belegen. ${ }^{36}$ Danach haben sich die Amtsträger des Ordens mit ihren Widersachern auf eine hochgestellte Persönlichkeit geeinigt und geschworen, deren Urteilsspruch anzuerkennen oder eine vorher festgelegte Strafe auf sich zu nehmen. Allein schon wegen des Ansehens des Schiedsrichters konnte der Streit durch dessen Spruch beigelegt werden. Des Schiedsgerichts bediente sich der Orden vor allem gegen Adlige. Sie war aber nicht die einzige Form, die er nutzte.

Der Deutsche Orden hatte sowohl in seinen hochmeisterlichen wie den deutschmeisterlichen Balleien Burgen und Herrschaften erworben oder ausgebildet. Die Burgen vor allem bildeten Ansatzpunkte für Streitigkeiten mit dem umliegenden Adel, aber auch den Landesherren. Jedoch wissen wir von fehdeähnlichen Streitigkeiten um die Burgen eigentlich wenig oder gar nichts. Die Kapfenburg wurde beispielsweise im 14. Jahrhundert von der Kommende Mergentheim erworben und entwickelte sich schließlich spätestens 1379 zu einer eigenen Kommende. ${ }^{37}$ Ein anderes Beispiel aus der Ballei Franken mag die Burg Virnsberg mit umfangreichem Besitz dienen. Sie wurde 1294 von den Burggrafen von Nürnberg

34 Weiß (wie Anm. 19), S. 355.

35 Vgl. W. Janssen, Bemerkungen zum Aufkommen der Schiedsgerichtsbarkeit am Niederrhein im 13. Jahrhundert, Jahrbuch des Kölnischen Geschichtsvereins 43 (1971), S. 79; J. Weitzel, Schiedsgericht, in: Lexikon des Mittelalters, Bd. 7, München 1995, Sp. 1454 f.; Reichert (wie Anm. 33), S. 93 f.

36 Codex diplomaticus ordinis sanctae Mariae Theutonicorum, Bd. 2, hrsg. v. J. H. Hennes, Mainz 1861, Nr. 59, 83; Regesten der Erzbischöfe von Köln, Bd. 3, 1, bearb. v. R. Knipping, Bonn 1913, Nr. 2160 und öfter.

37 Militzer, Von Akkon (wie Anm. 8), S. 326 f.; A. Gerlach, Chronik von Lauchbeim, Ellwangen 1907, S. 25-27; R. Kießling, Die Stadt und ihr Land (Städteforschung, Reihe A: Darstellungen 29), Köln-Wien 1989, S. 65. 
geschenkt und galt als eine der vielen Hauskommenden. Bereits 1299 ist ein erster Komtur bezeugt. ${ }^{38}$ Trotz aller Auseinandersetzungen vor allem des 15. Jahrhunderts blieb diese Burg von Fehden einzelner Adliger weitgehend verschont. ${ }^{39}$ Die Mainau war ein Sonderfall, weil sie auf einer Insel lag und kaum direkt anzugreifen war. Außerdem bot sie dem umliegenden Adel eine Heimstatt für nachgeborene Söhne, so dass dieser Adel nur ein geringes Interesse an Auseinandersetzungen mit der zur Ballei Elsaß-Burgund zählenden Kommende hatte. ${ }^{40}$ Zumindest was die Burgen betrifft, lassen sich die Belege häufen. Eroberungen oder Belagerungen der in den Händen des Deutschen Ordens gelangten Befestigungen sind nicht zu ermitteln. Das trifft auch für die Insel Mainau zu. Die Befestigungen waren eben keine Adelsburgen, sondern unterstanden dem besonderen Schutz der Kirche, weil sie als geistliche Einrichtungen galten. Im übrigen standen sie auch unter dem Schutz der weltlichen und geistlichen Landesherren. Vor allem die geistlichen Landesherren konnten mit dem Bann oder Interdikt drohen. Beide blieben trotz mancher Aufweichungen wirkungsvolle Strafandrohungen. ${ }^{41}$

Die Herrschaften des Ordens umfassten nicht nur Burgen, sondern wie Elsen auch Gerichtsbezirke oder gar Herrschaften mit kleineren Städten wie Mergentheim. Sicher hatten auch diese kleineren Herrschaften um die Anerkennung als selbständige Gebiete zu kämpfen. Ihre Gegner waren vor allem die Landesherren. In Mergentheim ist es außerdem nicht ohne Auseinandersetzungen des Ordens mit der Bürgerschaft abgegangen. Jedoch erwiesen sich die Einwohner als zu schwach gegenüber der Macht des Ordens. ${ }^{42}$ Zwar ist Mergentheim, das dortige Ordenshaus, dessen Komtur und der Deutschmeister während der zweiten Hälfte des 15. Jahrhunderts wiederholt in Fehden verwickelt worden, die aber nicht vom Orden ausgingen oder direkt gegen ihn gerichtet waren. ${ }^{43}$

38 Militzer, Von Akkon (wie Anm. 8), S. 326; Weiß (wie Anm. 19), S. 124-126.

39 G. Rechter, Das Land zwischen Aisch und Rezat (Schriften des Zentralinstituts für fränkische Landeskunde und allgemeine Regionalforschung an der Universität Erlangen-Nürnberg 20), Neustadt/Aisch 1981, S. $263 \mathrm{ff}$.

40 O. Feger, Die Deutsch-Ordens-Kommende Mainau (Schriften des Kopernikuskreises 2), Lindau, Konstanz 1958, S. 110 ff.; M. Diefenbacher, Das Urbar der Deutschordenskommende Mainau von 1394 (Veröffentlichungen der Kommission für geschichtliche Landeskunde in Baden-Württemberg, Reihe A: Quellen 39), Stuttgart 1989, S. 18 ff.; vgl. auch Militzer, Von Akkon (wie Anm. 8), S. 257.

41 Vgl. H. Zapp, Bann, in: Lexikon des Mittelalters, Bd. 1, München-Zürich 1980, Sp. 1416 f.; ders., Interdikt, in: ebd., Bd. 5, München-Zürich 1991, Sp. 466 f.

42 B. Klebes, Der Deutsche Orden in der Region Mergentheim im Mittelalter (Quellen und Studien zur Geschichte des Deutschen Ordens 58), Marburg 2002, S. 204 ff., 441 ff.

43 Ebd., S. $471 \mathrm{ff}$. 
Die Durchsetzung der eigenen Standpunkte oder das Festhalten an der eigenen Herrschaft war auch dadurch begünstigt, dass der Orden sich auf die Verwandten seiner Mitglieder stützen konnte. Es waren nicht die geistlichen Aufgaben, die der Orden erfüllte, sondern es war vor allem die Stellung als Reichsstand, wie Dieter J. Weiß formuliert hat, ${ }^{44}$ dazu eben auch die soziale Verflechtung oder das soziale Netz, das den Orden stützte.

Das führt wiederum zurück auf die Adelsfamilien, die Söhne in den Orden eintreten ließen. Nachdem der Orden 1244 Gerhard von Malberg in Montfort in Palästina zur Abdankung gezwungen hatte, waren dessen Angehörige damit nicht einverstanden. Trotz eines Schiedsgerichtsspruchs des Papstes blieb die Lage unklar. Nach Gerhards Tod führten dessen Söhne eine Fehde gegen den Orden angeblich um Geldforderungen. Erst 1260 konnte der Streit beigelegt werden. ${ }^{45}$ Nachdem Gottfried von Hohenlohe 1303 abgesetzt worden war, behielt er seinen Titel als Hochmeister und bereitete dem neu in das Amt gewählten Siegfried von Feuchtwangen in Venedig erhebliche Probleme. Gottfried stützte sich ebenfalls auf seine Verwandten. Allerdings wissen wir nicht, ob Gottfried oder seine Verwandten gegen den Orden mit Waffengewalt in Form einer Fehde vorgegangen sind. ${ }^{46}$

Zweifelsfrei haben die Verwandten Heinrichs von Löwenstein zusammen mit ihrem Ordensbruder eine Fehde gegen den Orden geführt. Löwenstein aus einem edelfreien Dynastengeschlecht hatte sich 1291 in der Kommende Marburg einkleiden lassen. ${ }^{47}$ Mit Hilfe seiner Verwandten führte er anschließend eine Fehde gegen das Deutschordenshaus Marburg, raubte auf offener Straße Getreide und fügte dem Orden weiteren Schaden zu. 1315 sollten er und zwei Jahre später sei-

44 Vgl. Weiß (wie Anm. 19), S. 366.

45 U. Arnold, Gerhard von Malberg, in: Die Hochmeister des Deutschen Ordens 1190-1994, hrsg. v. U. Arnold (Quellen und Studien zur Geschichte des Deutschen Ordens 40; Veröffentlichungen der Internationalen Historischen Kommission zur Erforschung des Deutschen Ordens 6), Marburg 1998, S. 22 f.; Regestenlijst der oorkonden van de landkommanderij Oudenbiezen en onderhorige kommanderijen, Tl. 1, hrsg. v. J. Grauwels, Brüssel 1966, Nr. 25, 48 f., 51 f.; Die Urkunden des Deutschordens-Centralarchivs zu Wien, hrsg. v. E. G. von Pettenegg, Prag-Leipzig 1887, Nr. 217 f., 365, 368, 377; Die Urkunden des Deutschordens-Zentralarchivs in Wien. Regesten, Bd. 1, hrsg. v. U. Arnold (Quellen und Studien zur Geschichte des Deutschen Ordens 60; Veröffentlichungen der Internationalen Historischen Kommission zur Erforschung des Deutschen Ordens 11), Marburg, 2006, Nr. 288, 290, 550, 552, 564.

46 K. Militzer, Gottfried von Hohenlohe, in: Hochmeister (wie Anm. 46), S. 48 f.; Hohenlohisches Urkundenbuch, Bd. 1, hrsg. v. K. Weller, Stuttgart 1899, Nr. 728 (45).

47 A. Wyß, Urkundenbuch der Deutschordens-Ballei Hessen, 3 Bde. (Hessisches Urkundenbuch, 1. Abt.) (Publicationen aus den K. Preußischen Staatsarchiven 3, 19, 73), Leipzig 1879-1899; hier: Bd. 1, Nr. 534; F. Schunder, Die von Löwenstein. Geschichte einer hessischen Familie, 2 Bde., Lübeck 1955, hier: Bd. 1, S. 13 ff. 
ne Verwandten dafür exkommuniziert werden. ${ }^{48}$ Nachdem 1318 Verhandlungen aufgenommen worden waren, stellte die Kommende in Marburg den Antrag, die Exkommunikation aufzuheben. ${ }^{49}$ Infolgedessen wurde die geistliche Strafe aufgehoben und Heinrich wieder in den Orden aufgenommen, erhielt aber zunächst kein Amt in der Ballei Hessen. Er war jedoch bereits 1325 Komtur von Regensburg, ${ }^{50}$ wurde 1326 Landkomtur von Thüringen und blieb es bis mindestens $1329,{ }^{51}$ bevor er schließlich 1332 doch noch Komtur von Marburg geworden ist..$^{52}$ Danach ist er $1332-1333$ als Komtur von Frankfurt, ${ }^{53}$ im Jahr darauf als Vogt des Deutschen Ordens von Kirchhain belegt, ${ }^{54}$ wurde 1335 Komtur von Mergentheim, ${ }^{55} 1338$ wieder Komtur von Regensburg ${ }^{56}$ und ist schließlich nach Preußen beordert worden, wo er 1339-1343 als einfacher Ordensbruder in der Marienburg lebte. ${ }^{57}$ Bald darauf wird er gestorben sein.

Die Tat des Ordensbruders Heinrich von Löwenstein hätte den Statuten nach eine Kerkerstrafe oder wenigstens eine Jahrbuße nach sich ziehen müssen. Aber mit mächtigen Familienangehörigen und einer einflussreichen Verwandtschaft im Rücken waren solche Strafen nicht durchzusetzen, jedenfalls nicht in den Balleien, die dem Einfluss der Adelsfamilien stärker als etwa in Preußen oder Livland ausgesetzt waren.

In den Balleien sind in einzelnen Urkunden weitere Hinweise auf solche Fehden aufzudecken. Beispielsweise hielten 1373 die Schöffen von Godesberg anlässlich der Verpachtung eines Weingartens durch den Deutschen Orden fest, dass

48 Wyß, Urkundenbuch (wie Anm. 48), Bd. 2, Nr. 283, 296, 306; Schunder (wie Anm. 48), Bd. 2, Nr. 128, 132 f.; Bd. 1, S. 192 f. Die Löwensteiner ertränkten übrigens 1337 einen Boten des Kardinals Anibaldo-Ceccano, weil dieser in Fritzlar die Übertragung einer Pfründe an den Kardinal durch den Papst verkünden wollte. Auf die Pfründe erhoben auch die Löwensteiner Ansprüche.

49 Wyß, Urkundenbuch (wie Anm. 48), Bd. 2, Nr. 331.

50 Weiß (wie Anm. 19), S. 473.

51 B. Sommerlad, Der Deutsche Orden in Thüringen (Forschungen zur thüringisch-sächsischen Geschichte 10), Halle/Saale 1931, S. 214.

52 U. Braasch-Schwersmann, Das Deutschordenshaus Marburg. Wirtschaft und Verwaltung einer spätmittelalterlichen Grundherrschaft (Untersuchungen und Materialien zur Verfassungs- und Landesgeschichte 11), Marburg 1989, S. 211.

53 J. Seiler, Der Deutsche Orden in Frankfurt (Quellen und Studien zur Geschichte des Deutschen Ordens 61), Marburg 2003, S. 505.

54 Wyß, Urkundenbuch (wie Anm. 48), Bd. 2, Nr. 599.

55 Klebes (wie Anm. 43), S. 699.

56 Weiß (wie Anm. 19), S. 444.

57 Preußisches Urkundenbuch, Bd. 3, 1, hrsg. v. M. Hein, Marburg 1944, Nr. 284,324, 342, 346, 349, 353, 357, 371, 410; Bd. 3, 2, hrsg. v. K. Conrad, Marburg 1961, Nr. 448, 464, 466, 479 , $486,488,490,492,541,544,550,554,557,596,615,621$. 
der aus der Kölner Kommende ausgetretene Arnold von Lülsdorf Schaden ausüben oder eine Fehde führen könne, weil Arnold von dem Weingarten für sich eine Leibzucht beanspruche. ${ }^{58}$ Derartige Fälle wird es mehr gegeben haben, wenn man die Urkunden sorgfältig daraufhin überprüft.

Der Fall Löwenstein bleibt dennoch spektakulär, zumal eine solche Fehde nicht oft mit Erfolg hätte abgeschlossen werden können. Jedoch war er im Laufe des Mittelalters keineswegs ein Einzelfall. Gerd von Mallinckrodt wurde 1468 als livländischer Marschall abgesetzt, leistete jedoch Widerstand und verschanzte sich schließlich in der livländischen Ordensburg Doblen. Er führte eine Fehde gegen den livländischen Ordenszweig. Auch nachdem er in seinen Abzug in das Reich eingewilligt hatte, gab er nicht auf, sondern bereitete seine Rückkehr mit Hilfe seiner Verwandten und anderer Fürsprecher wie den dänischen König vor. Das ist ihm auch gelungen. ${ }^{59}$ Der Fall Mallinckrodt soll uns nicht weiter beschäftigen, belegt aber, dass dergleichen auch in den folgenden Jahrhunderten und nicht nur in den Balleien von Erfolg gekrönt sein konnte. Jedoch möchte ich von vornherein betonen, dass die überwiegende Mehrzahl der Ordensbrüder die Wege eines Löwenstein oder Mallinckrodt nicht betreten haben.

Der Deutsche Orden und seine Amtsträger haben es tunlichst vermieden, in den Balleien gegen Christen eine Fehde anzuzetteln oder zu führen. Einmal verboten ihnen die Statuten die Gewaltanwendung gegen Christen, in deren Mitte sie lebten. Zweitens bevorzugten sie die Schiedsgerichtsbarkeit, weil sie sich von Anfang an an mächtige geistliche und weltliche Herren angeschlossen hatten, die sie als Schiedsrichter gewinnen konnten. Das soll nicht heißen, dass der Orden gar keine Fehden geführt habe oder von Adligen begonnene Fehden nicht habe aushalten müssen. Das Gegenteil ist vielfach bezeugt. Schließlich verfügte der Orden in seinen Balleien über Burgen und Herrschaftsrechte, die zu Fehden Anlass boten, was jedoch so gut wie gar nicht vorgekommen ist. Endlich haben Ordensbrüder selbst, wenn auch selten, Fehden gegen ihren eigenen Orden geführt, indem sie sich vorwiegend auf ihre Familien und andere Netzwerke ihrer adligen Verwandten stützen konnten. Jedoch ist zu betonen, dass solche Fehden eher die Ausnahme gebildet haben.

58 HAStK, St. Katharinen U1/439. Zu Disziplinarfällen vgl. auch K. Conrad, Zu Disziplinarfällen im Deutschen Orden unter Hochmeister Winrich von Kniprode, Preußenland 27 (1989), 1, S. $33 \mathrm{ff}$.

59 S. Neitmann, Von der Grafschaft Mark nach Livland (Veröffentlichungen aus den Archiven Preußischer Kulturbesitz, Beiheft 3), Köln-Weimar-Wien 1993, S. 410 ff. 